\title{
Aquisição de habilidade em sutura laparoscópica
}

\author{
Acquisition of skill in laparoscopic suture \\ Hamilton Cezar Rocha Garcia', Wescley Miguel Pereira da Silva², Andressa Miléo Ferraioli Silva', Brisa da Silva Lopes², \\ Daniel Haber Feijó', Mariana Albuquerque Dórea', Luiz Claudio Lopes Chaves', Marcus Vinicius Henriques Brito
}

Resumo Objetivo: Avaliar aquisição de habilidade de residentes de Cirurgia Geral em modelo de treinamento de sutura videocirúrgica. Método: Selecionaram-se randomicamente 7 preceptores com experiência comprovada na técnica videolaparoscópica (Grupo P), 7 residentes do $1^{\circ}$ ano (Grupo R1) e 7 residentes do $2^{\circ}$ ano (Grupo R2) dentre os serviços de residência médica em Cirurgia Geral de Belém do Pará. Todos foram submetidos a 4 sessões práticas de treinamento em sutura videolaparoscópica utilizando modelos de fibra de vidro e etil vinil acetato (E.V.A.) do Centro de Treinamento de Laparoscopia do Centro Hospitalar Jean Bitar, para aferição do tempo necessário para a realização dos 5 nós por cada indivíduo do estudo. Os dados obtidos foram analisados pelos testes Friedman e ANOVA um critério (complementado pelo teste de TUKEY). Resultados: Entre os membros do Grupo R1 houve ganho estatisticamente significante entre a $1^{\mathrm{a}}$ e $3^{\mathrm{a}}$ semanas, entre a $1^{\mathrm{a}}$ e $4^{\mathrm{a}}$ e entre a $2^{\mathrm{a}}$ e $4^{\mathrm{a}}$. Nos membros do grupo R2 houve ganho estatisticamente significante entre a $1^{\mathrm{a}}$ e $2^{\mathrm{a}}$, entre a $1^{\mathrm{a}}$ e $3^{\mathrm{a}}$ e entre a $1^{\mathrm{a}}$ e $4^{\mathrm{a}}$. Já no grupo $\mathrm{P}$, houve ganho estatisticamente significante entre a $1^{\mathrm{a}}$ e $4^{\mathrm{a}}$ e entre a $2^{\mathrm{a}}$ e $4^{\mathrm{a}}$. Na análise intergrupos, houve igualdade entre os tempos dos grupos R1 e R2 nas 4 semanas e estes obtiveram tempos maiores que o Grupo P em todas as quatro semanas com $p=0,01$. Conclusão: Houve diminuição do tempo de confecção da sutura videocirúrgica, no modelo de treinamento proposto.

Descritores: cirurgia geral; educação médica; laparoscopia.

Summary Purpose: To evaluate skill acquisition of residents of General Surgery in a model of surgical suture training. Method: Seven preceptors with proven experience in videolaparoscopic technique (Group P), 7 first-year residents (Group R1) and 7 second-year residents (Group R2) were randomly selected from among the medical residency services in General Surgery of Belém/Pa. All of them underwent 4 practical sessions of videolaparoscopic suture training using fiberglass and ethyl vinyl acetate (EVA) models of the Laparoscopic Training Center of the Jean Bitar Hospital Center, in order to assess the time required to perform the 5 knots per each study subject. The data obtained were analyzed by the Friedman tests and one ANOVA criterion (complemented by the TUKEY test). Results: Among the members of the R1 group there was a statistically significant gain between the 1st and 3rd weeks, between the 1st and 4th and between the 2 nd and $4^{\text {th }}$. Among the members of the R2 group there was a statistically significant gain between the 1st and 2nd, 1st and 3rd and between 1st and 4th. In group P, there was a statistically significant gain between the 1 st and 4 th and between the 2 nd and 4 th. In the intergroup analysis, there was equality between the times of the R1 and R2 groups at the 4 weeks, and these times had times greater than the Group P in all four weeks with $\mathrm{p}=0.01$. Conclusion: There was a decrease in the length of time of the laparoscopic suture, in the proposed training model.

Keywords: general surgery; education, medical; laparoscopy.

'Universidade do Estado do Pará - UEPA, Belém, PA, Brasil

¿Universidade Federal do Pará - UFPA, Belém, PA, Brasil

Fonte de financiamento: Nenhuma.

Conflitos de interesses: Os autores declaram não haver conflitos de interesse.

Recebido: Junho 11, 2018

Aceito: Julho 06, 2018

Trabalho realizado no Mestrado de Pesquisa e Cirurgia Experimental, ligado ao Laboratório de Cirurgia Experimental da Universidade do Estado do Pará - UEPA, Belém, PA, Brasil.

Copyright Garcia et al. Este é um artigo publicado em acesso aberto (Open Access) sob a licença Creative Commons Attribution, que permite uso, distribuição e reprodução em qualquer meio, sem restrições desde que o trabalho original seja corretamente citado. 


\section{Introdução}

Para aquisição de habilidades cirúrgicas laparoscópicas, é primordial que haja o treinamento adequado do profissional, de preferência em simuladores de boa qualidade ${ }^{1}$. Quando treinados, os profissionais têm desempenho intraoperatório superior em habilidades cirúrgicas complexas quando comparados a indivíduos sem treinamento prévio ${ }^{2,3}$. Em relação ao método de treinamento, é imprescindível que leve em consideração a preferência pelo tipo de modelo para estudo. Em se tratando de residentes de cirurgia geral, é notória preferência por modelos de vídeo-treinamento ${ }^{4-6}$. $O$ treinamento em videocirurgia deve ser iniciado o mais breve possível ${ }^{7}$.

O treinamento cirúrgico tem sido tradicionalmente um dos métodos de aprendizagem, onde o estagiário aprende a realizar uma cirurgia sob supervisão de um cirurgião treinado. Este é demorado, dispendioso, e de eficácia variável. O treinamento usando um modelo de caixa de simulador é uma opção para complementar o treinamento padrão, contudo a supervisão por um profissional capacitado ainda é indispensável ${ }^{8}$.

Devido aos aspectos éticos e legais envolvidos no manuseio de cadáveres e animais, os simuladores sintéticos surgem como alternativa para o ensino-aprendizagem de técnicas de sutura e simulação de procedimentos $^{9,10}$. Dentre os modelos utilizados, vale destacar o modelo EVA (etileno-vinil-acetato)l o qual permite que os alunos adquiram habilidade de sutura mais rapidamente quando comparadas ao modelo tradicional de ensino ${ }^{10,11}$.

Além das técnicas de sutura, é importante que se busque desenvolver uma técnica adequada de fundoplicatura para melhor manejo do espaço tridimensional a ser operado, uma vez que a perda da sensibilidade tátil compartimental é um dos desafios da videocirurgia. Para treinamento de fundoplicatura, os estudos apotam que a técnica de Nissen é a de marcada preferência ${ }^{11}$. A cirurgia de Nissen por videolaparoscopia requer a capacidade de executar uma operação em vários planos em um ambiente tridimensional com feedback visual bidimensional. A aquisição destas habilidades e a necessidade de adquirir facilidade utilizando novos instrumentos, técnicas de sutura, e novos dispositivos tecnológicos são desafios na realização de uma operação segura e eficaz ${ }^{12}$.

A curva de aprendizado para a fundoplicatura videolaparoscópica tem impacto estatisticamente significativo nos resultados clínicos em pacientes, uma vez que residentes treinados e supervisionados por cirurgiões experientes de forma contínua têm resultados cirúrgicos semelhantes a estes últimos e apresenta melhor retenção de habilidades, de forma mais eficiente e eficaz, contribuindo, dessa forma, para a melhor qualidade de seus serviços prestados ${ }^{13,14}$.

Sendo assim, buscou-se avaliar a aquisição de habilidades cirúrgicas em modelo modificado de treinamento para cirurgia videolaparoscópica em treinamento com residentes dos Serviços de Residência em Cirurgia Geral de Belém do Pará.

\section{Método}

Após aprovação do comitê de ética (número do parecer: 1.116.689), foram realizados os treinamentos em videocirurgia. Foram selecionados para o treinamento 7 preceptores com experiência comprovada em videolaparoscopia (mínimo de 10 anos), 7 residentes do $1^{\circ}$ ano e 7 residentes do $2^{\circ}$ ano selecionados randomicamente dos serviços de residência médica em Cirurgia Geral de Belém.

Cada grupo de sete indivíduos foi assim distribuído: Grupo P (controle) - Preceptores; Grupo R1 Residentes do $1^{\circ}$ ano (R1); Grupo R2 - Residentes do $2^{\circ}$ ano (R2).

Os indivíduos dos Grupos P, R1 e R2 foram submetidos a uma sessão de vídeo-aula ${ }^{15}$ teórica que demonstrou a técnica de sutura videolaparoscópica realizada nesse estudo, seguida de sessão de treino livre de 30 minutos. Após a vídeo-aula e a sessão de treino livre foram realizadas 4 sessões (com intervalo de 7 dias entre elas - Dias 1, 8, 15 e 22) de prática de sutura videolaparoscópica em modelos de fibra de vidro (Figura 1) existentes no Centro de Treinamento de Laparoscopia do Centro Hospitalar Jean Bitar.

Nos modelos de fibra de vidro foram realizados cinco nós, com três semi-nós cada (Figura 2), em modelos de sutura feitos de Etil Vinil Acetato (E.V.A.) (Figura 3) simulando assim a quantidade de nós realizados em uma fundoplicatura à Nissen que é caracterizada pela realização de cinco nós sendo dois para aproximação dos pilares diafragmáticos e três para a confecção da válvula gástrica anti-refluxo.

Nas quatro sessões práticas foi aferido o tempo necessário para cada grupo realizar os cinco nós sem limite de tempo. 


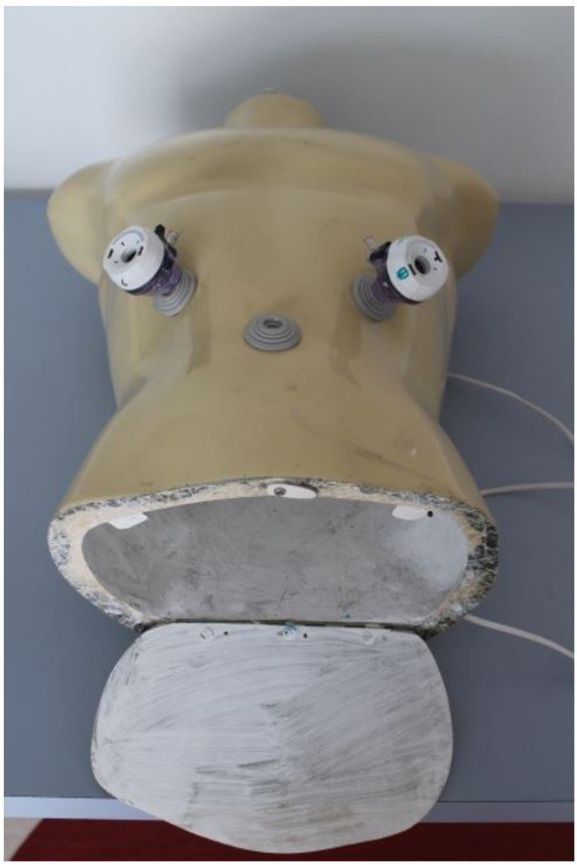

Figura 1. Modelo de fibra de vidro. Fonte: Protocolo da pesquisa.

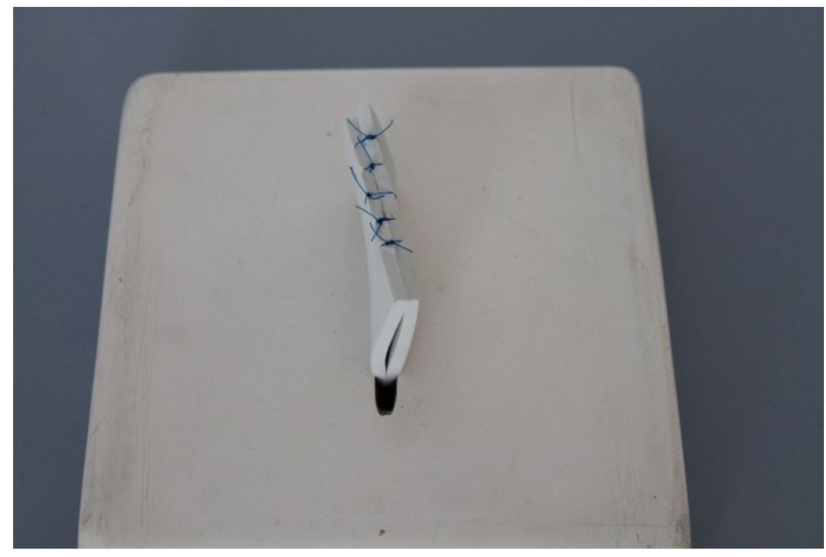

Figura 2. Modelo de E.V.A. após os cinco nós realizados.

Fonte: Protocolo da pesquisa.

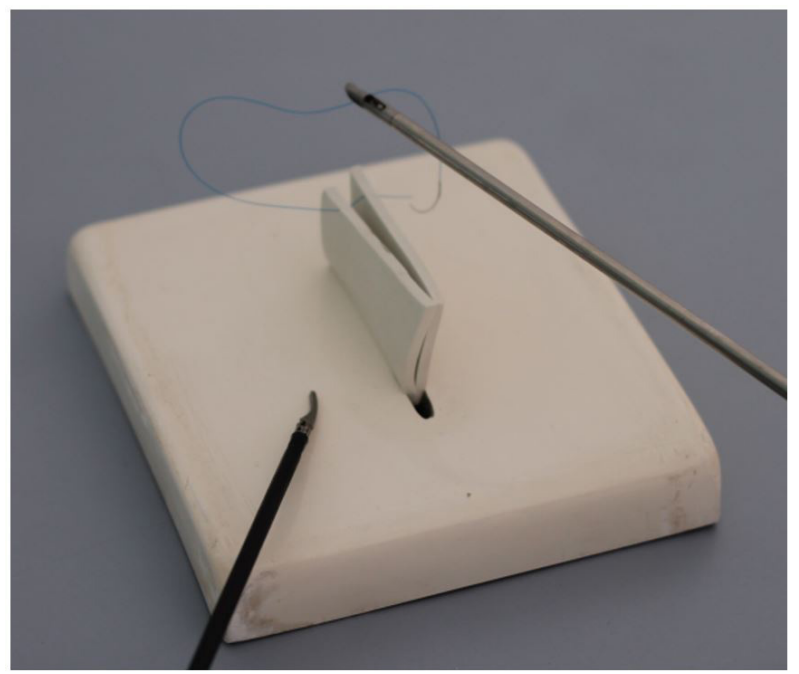

Figura 3. Modelo de sutura feito de E.V.A.

Fonte: Protocolo da pesquisa. 
O fio utilizado para a confecção dos nós foi o Vicryl ${ }^{\circledR}$ 2-0.

Os dados obtidos de tempo de confecção dos nós em cada uma das 4 sessões de treinamento prático foram compilados em planilha do Microsoft Excel $2010^{\circledR} \mathrm{e}$ analisados estatisticamente usano-se o programa BioEstat 5.3 através dos testes de Friedman (intraagrupos) e ANOVA um critério complementado pelo Teste de TUKEY (intergrupos) utilizando-se como nível de significância $\alpha=0,05$.

\section{Resultados}

A casuística do Grupo R1 apresentou diferença significativa $(\mathrm{p}<0,05)$ quando comparadas a $1^{\mathrm{a}} \mathrm{e} 3^{\mathrm{a}}$ semanas, $1^{\mathrm{a}}$ e $4^{\mathrm{a}}$ semanas e ao se analisar a $2^{\mathrm{a}}$ e $4^{\mathrm{a}}$ semanas. Entretanto, não se observou diferenças estatísticas significativas quando comparadas $1^{\mathrm{a}}$ e $2^{\mathrm{a}}$ semanas, $2^{\mathrm{a}}$ e $3^{\mathrm{a}}$ semanas e $3^{\mathrm{a}}$ e $4^{\mathrm{a}}$ semanas.

No que tange o Grupo R2, houve diferença estatística significativa $(\mathrm{p}<0,05)$ quando analisadas a $1^{a}$ semana em relação às semanas 2,3 e 4 . Diferença esta, não observada ao se comparar a $2^{a}$ semana com a $3^{\mathrm{a}}$ e $4^{\mathrm{a}}$ semanas e ao se avaliar a $3^{\mathrm{a}}$ com a $4^{\mathrm{a}}$ semanas, separadamente.

Em contraste ao Grupo P que evidenciou mudanças estatisticamente significativas apenas ao se comparar a semana $1 \mathrm{com}$ a 4 e ao se relacionar a $2^{\mathrm{a}} \mathrm{com}$ a $4^{\mathrm{a}}$ semanas. Não havendo mudanças significativas ao se analisar a $1^{\mathrm{a}} \operatorname{com}$ a $2^{\mathrm{a}}$ e $3^{\mathrm{a}}$ semanas e ao se avaliar a $3^{\mathrm{a}} \operatorname{com}$ a $2^{\mathrm{a}}$ e a $4^{\mathrm{a}}$ semanas.

$\mathrm{Na}$ análise intergrupos, desde a $1^{\mathrm{a}}$ até a $4^{\mathrm{a}}$ semana, os grupos R1 e R2 obtiveram desempenhos estatisticamente iguais ( $p>0,05)$, sendo, também, o desempenhos desses dois grupos inferior aos apresentados pelos integrantes do grupo $\mathrm{P}(\mathrm{p}<0,01)$ (Figura 4).

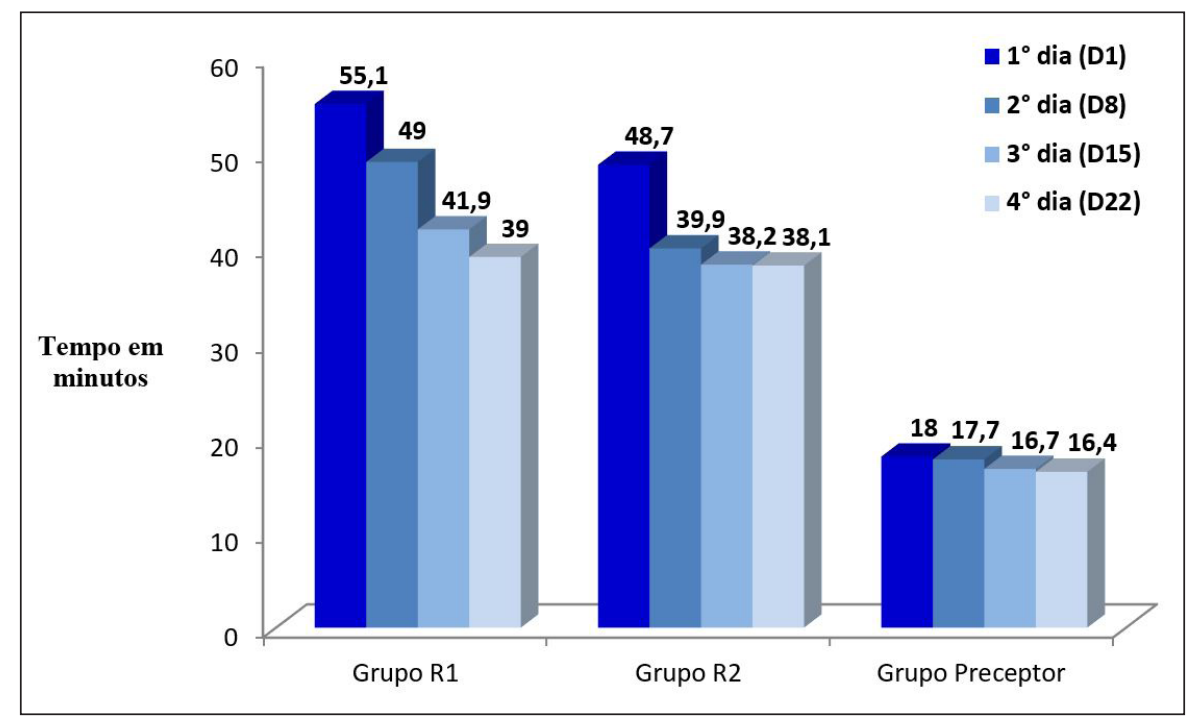

Figura 4. Evolução tempo de sutura de acrodo com as semanas.

Fonte: Protocolo da pesquisa.

\section{Discussão}

Muitas mudanças na forma como os jovens cirurgiões são ensinados a operar ocorreram nos últimos $\operatorname{anos}^{16}$.

A capacidade de realizar nós e suturas é fundamental na área cirúrgica, tanto em procedimentos convencionais (abertos), quanto nos procedimentos laparoscópicos ${ }^{1,17}$. Nestes últimos, o emprego de instrumentais cirúrgicos diferenciados, fixos e a visualização apenas em duas dimensões, torna tal tarefa extremamente trabalhosa e árdua em seus aspectos técnicos, podendo ser frustrante e demorada, mesmo para cirurgiões laparoscopistas experientes ${ }^{5}$.

O treinamento extenso em simulador é uma das formas mais eficazes de futuros cirurgiões vencerem a bem estabelecida curva de aprendizado para tais procedimentos ${ }^{18}$, conjuntamente com a ampla gama de operações já realizadas utilizando técnicas minimamente invasivas, futuros cirurgiões têm a oportunidade de desenvolver essas habilidades no início de suas carreiras, havendo evidências que cirurgiões mais novos 
podem utilizar os conhecimentos de quem foi pioneiro nas técnicas para dominá-las mais rápido do que cirurgiões mais experientes ${ }^{1}$.

A participação de preceptores com experiência de mais de 10 anos na presente pesquisa, visou a utilização dos mesmos para definição de tempos padrão a serem comparados com os residentes de $1^{\circ}$ e $2^{\circ}$ anos. $\mathrm{Na}$ análise intragrupo de seu treinamento, observou-se que mesma com extensa experiência prévia, houve uma melhora no seu desempenho após as 4 semanas de treinamento, estando de acordo com a literatura que recomenda o treinamento constante de tais profissionais, tendo sua eficácia comprovada para treinamentos no mínimo mensais. Não se pode descartar a hipótese de adaptação ao modelo de treinamento introduzido àqueles com maior prática in vivo, porém o esperado seria uma melhora da primeira para a segunda semana devido a adaptação, o que não foi evidenciando pelos resultados encontrados.

Esta última situação citada ocorreu com os residentes de segundo ano, os quais apresentaram apenas uma melhora da primeira para as três semanas subsequentes, as quais mantiveram entre si igualdade estatística. Tais residentes já haviam sido expostos previamente a certos modelos de treinamento em videocirurgia, levando-os apenas a melhora devido à adaptação ao modelo proposto pelo estudo, em contraste com estudo de Brown ${ }^{13}$ que evidenciou uma melhora nos indivíduos já expostos a treinamento prévio, devido esses, tradicionalmente, encontrarem-se já mais avançados na curva de aprendizado.

Já os residentes de primeiro ano, sem treinamento prévio algum, foram os que apresentaram ganhos mais expressivos no presente estudo. Na analise intragrupo, pode-se notar uma melhora da primeira para a terceira e quarta semanas e da segunda para a quarta, o que evidencia a necessidade de pelo menos duas semanas para tais indivíduos começarem a ter ganhos estatísticos no desempenho de sua técnica. Tal achado é fundamental para o desenvolvimento de futuros cronogramas de treinamento e também para uma melhor avaliação desses residentes pelos seus preceptores no processo de aprendizado, contribuindo para o melhor treinamento nos programas de residência médica.

$\mathrm{Na}$ análise intergrupo, pode-se evidenciar a já esperada soberania dos preceptores em todas as semanas sobre ambos os grupos de residentes, sugerindo assim uma necessidade de tempo muito maior de treinamento para equiparação da técnica de sutura em cirurgia minimamente invasiva. Relevante também nessa análise é o fato que em todas as semanas os residentes de primeiro ano tiveram desempenho estatisticamente igual aos de segundo $\mathrm{ano}^{19}$, fugindo do melhor desempenho esperado pelos R2s, inclusive na segunda semana, tornando-se claro que a brevidade no início dos treinamentos é de extrema importância, uma vez que ao final de quatro semanas já existia uma equiparação entre os grupos R1 e R2.

\section{Conclusão}

Assim sendo, observou-se que houve ganho de habilidade no treinamento em videocurgia, no tocante à diminuição do tempo de confecção da sutura videocirúrgica, no modelo de treinamento proposto.

\section{Referências}

1. Cavalini WLP, Claus CM, Dimbarre D, Cury Fo AM, Bonin EA, Loureiro MP, et al. Development of laparoscopic skills in medical students naive to surgical training. Einstein (Sao Paulo). 2014;12(4):467-72. http://dx.doi.org/10.1590/S1679-45082014AO3237. PMid:25628198.

2. Van Sickle KR, Ritter EM, Baghai M, Goldenberg AE, Huang IP, Gallagher AG, et al. Prospective, randomized, double-blind trial of curriculum-based training for intracorporeal suturing and knot tying. J Am Coll Surg. 2008;207(4):560-8. http://dx.doi.org/10.1016/j. jamcollsurg.2008.05.007. PMid:18926460.

3. Harold KL, Matthews BD, Backus CL, Pratt BL, Heniford BT. Prospective randomized evaluation of surgical resident proficiency with laparoscopic suturing after course instruction. Surg Endosc. 2002;16(12):1729-31. http://dx.doi.org/10.1007/s00464-002-8832-8. PMid:12140636.

4. Palter VN, Orzech N, Aggarwal R, Okrainec A, Grantcharov TP. Resident perceptions of advanced laparoscopic skills training. Surg Endosc. 2010;24(11):2830-4. http://dx.doi.org/10.1007/s00464-010-1561-5. PMid:20428895.

5. Pattaras JG, Smith GS, Landman J, Moore RG. Comparison and analysis of laparoscopic intracorporeal suturing devices: preliminary results. J Endourol. 2001;15(2):187-92. http://dx.doi.org/10.1089/089277901750134566. PMid:11325091.

6. Leonardi PC, Zilberstein B, Jacob CE, Yagi O, Cecconello I. Nós e suturas em vídeo-cirurgia: orientações práticas e técnicas. ABCD Arq Bras Cir Dig. 2010;23(3):200-5. http://dx.doi.org/10.1590/S0102-67202010000300014.

7. Subramanian A, Bakaeen FG. The case for teaching basic surgical skills in medical school. J Surg Res. 2012;176(2):406-8. http://dx.doi. org/10.1016/j.jss.2011.05.053. PMid:21764068. 
8. Gurusamy KS, Nagendran M, Toon CD, Davidson BR. Laparoscopic surgical box model training for surgical trainees with limited prior laparoscopic experience. Cochrane Database Syst Rev. 2014;1(3):CD010478. PMid:24585169.

9. Bastos EM, Silva RDP. Proposal of a synthetic ethylene-vinyl acetate bench model for surgical foundations learning. Suture training. Acta Cir Bras. 2011;26(2):149-52. http://dx.doi.org/10.1590/S0102-86502011000200014. PMid:21445480.

10. Denadai R, Saad-Hossne R, Oshiiwa M, Bastos EM. Training on synthetic ethylene-vinyl acetate bench model allows novice medical students to acquire suture skills. Acta Cir Bras. 2012;27(3):271-8. http://dx.doi.org/10.1590/S0102-86502012000300012. PMid:22460260.

11. Rocha JRM, Cecconello I, Félix VN, Sallum RAA, Oliveira MA, Gama-Rodrigues J. Aspectos clínicos, anatômicos e técnicos atuais no tratamento cirúrgico da doença do refluxo gastroesofágico. Rev Bras Videocir. 2004;2(4):201-5.

12. Botden SM, Christie L, Goossens R, Jakimowicz JJ. Training for laparoscopic Nissen fundoplication with a newly designed model: a replacement for animal tissue models? Surg Endosc. 2010;24(12):3134-40. http://dx.doi.org/10.1007/s00464-010-1104-0. PMid:20526629.

13. Brown CN, Smith LT, Watson DI, Devitt PG, Thompson SK, Jamieson GG. Outcomes for trainees vs experienced surgeons undertaking laparoscopic antireflux surgery - is equipoise achieved? J Gastrointest Surg. 2013;17(7):1173-80. http://dx.doi.org/10.1007/s11605-0132211-8. PMid:23653375.

14. Van Bruwaene S, Schijven MP, Miserez M. Maintenance training for laparoscopic suturing: the quest for the perfect timing and training model: a randomized trial. Surg Endosc. 2013;27(10):3823-9. http://dx.doi.org/10.1007/s00464-013-2981-9. PMid:23660721.

15. Tolerton SK, Hugh TJ, Cosman PH. The production of audiovisual teaching tools in minimally invasive surgery. J Surg Educ. 2012;69(3):404-6. http://dx.doi.org/10.1016/j.jsurg.2011.10.017. PMid:22483144.

16. Fingerhut A, Veyrie N, Millat B, Leandros E. Education and teaching laparoscopic surgery in Europe: present constraints and role of the European Association for Endoscopic Surgery. Cir Cir. 2011;79(1):46-52. PMid:21477518.

17. Purim KSM, Santos LDS, Murara GT, Maluf EMCP, Fernandes JW, Skinovsky J. Avaliação de treinamento cirúrgico na graduação de medicina. Rev Col Bras Cir. 2013;40(2):152-6. http://dx.doi.org/10.1590/S0100-69912013000200012. PMid:23752643.

18. Santos EG, Bravo No GP. Curva de aprendizado e lesões iatrogênicas em colecistectomias videolaparoscópicas. Rev Col Bras Cir. 2010;37(3):184-9. http://dx.doi.org/10.1590/S0100-69912010000300005. PMid:21079890.

19. Mahmoud A, Ward C, Padmesh H, Daher M. Safety and feasibility of the teaching assistant role of senior surgical residents: a prospective randomized study. J Surg Educ. 2012;69(2):249-52. http://dx.doi.org/10.1016/j.jsurg.2011.11.002. PMid:22365875.

\author{
Autor correspondente \\ Hamilton Cezar Rocha Garcia \\ Universidade do Estado do Pará - UEPA, CCBS II, Faculdade de Medicina, \\ Laboratório de Cirurgia Experimental, Tv. Perebebuí, 2623 - Marco, \\ CEP 66087-662, Belém, PA \\ Tel.: (91) 981158790 \\ E-mail: doutorhamilton@yahoo.com.br
}

Informação sobre os autores

HCRG é mestre em Cirurgia e Pesquisa Experimental pela Universidade do Estado do Pará (UEPA); possui residência médica em Cirurgia Geral pelo Hospital Ophir Loyola, docente dos cursos de Medicina da Faculdade Metropolitana da Amazônia (FAMAZ) e Universidade do Estado do Pará (UEPA).

WMPS é acadêmico de Medicina da Universidade Federal do Pará (UFPA); estagiário do Laboratório de Cirurgia Experimental.

BSL é acadêmica de Medicina da Universidade Federal do Pará (UFPA), estagiária do Laboratório de Habilidades Médicas.

AMFS é acadêmica de Medicina da Universidade do Estado do Pará (UEPA); estagiária do Laboratório de Cirurgia Experimental.

DHF é médico pela Universidade do Estado do Pará (UEPA); estagiário do Laboratório de Cirurgia Experimental.

MAD é acadêmica de Medicina da Universidade do Estado do Pará (UEPA), estagiária do Laboratório de Cirurgia Experimental.

LCLC é professor doutor do Internato de Clínica Cirúrgica, Universidade do Estado do Pará (UEPA).

MVHB é professor titular e coordenador do Mestrado Profissional em Cirurgia e Pesquisa Experimental e do Laboratório de Cirurgia Experimental, Universidade do Estado do Pará (UEPA).

\title{
Contribuição dos autores
}

HCRG: foi responsável pela concepção e delineamento, aquisição de informações clínicas e interpretação dos casos abordados; WMPS foi responsável formatação final do artigo, organização da equipe e elaboração do resumo; BSL, AMFS, DHF e MAD foram responsáveis pela avaliação dos resultados e preparação do artigo; LCLC e MVHB foram responsáveis pela orientação técnico-científica e avaliação do trabalho.

Todos os autores leram e aprovaram a versão final submetida ao Pará Research Medical Journal. 